ACCEPted to ApJ

Preprint typeset using LATEX style emulateapj v. 08/22/09

\title{
PLANET FORMATION AROUND STARS OF VARIOUS MASSES: HOT SUPER-EARTHS
}

\author{
GRANT M. KENNEDY \\ Research School of Astronomy and Astrophysics, Mt Stromlo Observatory, Australian National University, ACT 2611, Australia \\ AND \\ SCOTT J. KENYON \\ Smithsonian Astrophysical Observatory, Cambridge, MA 02138, USA \\ Accepted to $\mathrm{ApJ}$
}

\begin{abstract}
We consider trends resulting from two formation mechanisms for short-period super-Earths: planetplanet scattering and migration. We model scenarios where these planets originate near the snow line in "cold finger" circumstellar disks. Low-mass planet-planet scattering excites planets to low periastron orbits only for lower mass stars. With long circularisation times, these planets reside on long-period eccentric orbits. Closer formation regions mean planets that reach short-period orbits by migration are most common around low-mass stars. Above $\sim 1 M_{\odot}$, planets massive enough to migrate to close-in orbits before the gas disk dissipates are above the critical mass for gas giant formation. Thus, there is an upper stellar mass limit for short-period super-Earths that form by migration. If disk masses are distributed as a power law, planet frequency increases with metallicity because most disks have low masses. For disk masses distributed around a relatively high mass, planet frequency decreases with increasing metallicity. As icy planets migrate, they shepherd interior objects toward the star, which grow to $\sim 1 M_{\oplus}$. In contrast to icy migrators, surviving shepherded planets are rocky. Upon reaching short-period orbits, planets are subject to evaporation processes. The closest planets may be reduced to rocky or icy cores. Low-mass stars have lower EUV luminosities, so the level of evaporation decreases with decreasing stellar mass.
\end{abstract}

Subject headings: planetary systems: formation - planetary systems: protoplanetary disks

\section{INTRODUCTION}

With nearly 300 known extra-Solar planets, there are now several clear correlations between the properties of the planets and their host stars. The most well known trend is the increase in gas giant frequency with host star metallicity (e.g. Fischer \& Valenti 2005). Recent radial velocity surveys suggest that giant planet frequency also increases with stellar mass (Johnson et al. 2007).

These trends provide tests of planet formation theories. In the core accretion model for example, gas giant planets form by coagulation of small planetesimals near the "snow line" that separates rocky and icy regions in a circumstellar disk. Once icy protoplanets reach a critical core mass, they accrete gas rapidly (Pollack et al. 1996). Cores benefit from extra planet building material provided by enhanced metallicities and an increase in disk masses with stellar mass. The model is thus consistent with current observations (Ida \& Lin 2004, 2005; Kennedy \& Kenyon 2008).

Gravitational instability (GI) is an alternative formation mechanism for gas giant planets, where a relatively massive disk cools enough to fragment into Jupiter-mass clumps. Although GI operates over a wide range of stellar masses, there is still debate about predicted trends with metallicity (Durisen et al. 2007). Given observational biases in the current sample of extra-Solar planets, GI cannot be ruled out as a formation mechanism (Durisen et al. 2007).

Core accretion and GI models suggest that short-

Electronic address: grant@mso.anu.edu.au Electronic address: kenyon@cfa.harvard.edu period "hot Jupiters" reside too close to their parent stars to have formed in situ. Thus, these planets must migrate or scatter from more distant formation regions to arrive at their final orbits (Lin et al. 1996; Rasio \& Ford 1996). A combination of these two mechanisms probably operates to produce the observed distribution of extra-Solar giant planets. Scattering can reproduce most of the observed eccentricity distribution, but has trouble accounting for planets in circular orbits at distances too far from their host stars for tidal circularisation (Ford \& Rasio 2007). Migration theories can explain systems with planets in meanmotion resonances (Lee \& Peale 2002), but they may not reproduce the observed eccentricity distribution (e.g. Tremaine \& Zakamska 2004).

With the discovery of the first super-Earths in relatively short period orbits, migration and scattering remain possible mechanisms for planets to reach these radii (Brunini \& Cionco 2005; Terquem \& Papaloizou 2007; Raymond et al. 2007). However, the discovery of lowmass planets in systems already harbouring giant planets suggests new formation mechanisms (Zhou et al. 2005). Because these models require gas giants, they predict trends with metallicity and stellar mass for low-mass planets similar to those for giant planets. Though some low-mass planets may have formed with help from giant planets, a flatter metallicity distribution (Udry et al. 2007) and the absence of giant planets in some low-mass planet systems (e.g. Gl581 \& GJ674, Bonfils et al. 2005, 2007) indicate other formation mechanisms.

Here, we consider trends that may arise in forming short-period and/or transiting icy/rocky planets in sys- 
tems with no gas giants, over a range of stellar masses. The close-in planets that form are therefore the most massive in the planetary system. We first cover some background in $\S 2$. In $\S 3$ we use $n$-body simulations to show that $10 M_{\oplus}$ planet-planet scattering is unlikely to result in transiting planets for all but the lowest mass stars. With long circularisation timescales, planets in these systems are hard to detect. We consider migration scenarios using analytic, semi-analytic and $n$-body models in $\S 4$. With migration, short-period low-mass planets most likely form around low-mass stars. Above a certain stellar mass, it is hard to form any short-period planets without giant atmospheres. Trends with metallicity depend on the disk mass distribution. Migration to short-period orbits results in significant amounts of material being shepherded inward, which affects the final structure of these systems. We discuss our results, subsequent planetary evolution, and conclude in $\S 5$.

\section{BACKGROUND}

\subsection{General Picture}

Planets form in circumstellar disks. Therefore disk structure plays a key role in setting the final configuration of planetary systems. In most planet formation models, disk structure is characterised by an outwardly decreasing radial surface density profile. This profile usually includes an increase in surface density at the "snow line," where the temperature becomes low enough for water to freeze.

Planets form by accumulating solids in the disk. Therefore the expected increase in surface density at the snow line is often associated with the formation of gas giants like Jupiter. Forming Jupiter requires the relatively rapid growth of a $\sim 5-10 M_{\oplus}$ icy core, followed by a period of gas accretion (Pollack et al. 1996). Gas accretion must be complete before the gas disk disperses in $~ 3 \mathrm{Myr}$ (e.g. Haisch et al. 2001). In the minimum mass Solar nebula model (MMSN, Weidenschilling 1977; Hayashi 1981), forming the icy core rapidly requires factor of 5-10 surface density enhancements relative to the terrestrial region (Lissauer 1987; Pollack et al. 1996; Thommes et al. 2003). This factor is larger than the factor of 2-3 enhancements expected from Solar abundances (Asplund et al. 2005), or suggested by comet composition (Küppers et al. 2005), and the factor of $\sim 4$ derived in the original MMSN model (Hayashi 1981).

The need for larger surface density enhancements inspired "cold finger" disk models, which produce much larger snow line enhancements in a relatively narrow $(\lesssim \mathrm{AU})$ radial region near the snow line (Stevenson \& Lunine 1988; Cuzzi \& Zahnle 2004). In this picture, a circumstellar disk has an initial equilibrium state with the water vapour (ice) concentration decreasing (increasing) beyond the snow line. As the disk diffuses and advects, water continually condenses from gas passing beyond the snow line, thus enhancing the local surface density of solids, and removing vapour phase water from the inner disk. Sublimation of planetesimals that drift inside the condensation radius by gas drag enhances this effect: the surface density beyond the snow line increases when water vapour from the sublimated planetesimals diffuses back outside the snow line (Cuzzi \& Zahnle 2004).
The first cold finger models predict a factor of $\sim 10$-100 increase in the surface density of icy material in a relatively narrow region near the snow line (Stevenson \& Lunine 1988; Cuzzi \& Zahnle 2004). Using a more complex global disk model, Ciesla \& Cuzzi (2006) suggest surface density enhancements closer to 10 than 100. In their simulations, the enhancement regions are several AU wide at half the maximum planetesimal surface density.

The main differences expected for planet formation models using cold finger instead of MMSN disks are threefold. Due to the nature of the surface density enhancement: (i) fewer large planets form, (ii) large planets form in relatively low-mass disks, and (iii) planets form from material with much higher ice/rock ratios. In addition, material lost to inward planetesimal drift by gas drag (Thommes et al. 2003) may be returned to the cold finger region, allowing continued growth. Reducing the removal of drifting planetesimals enhances growth rates, and allows formation of more massive icy planets.

\subsection{Mathematical Formalism}

In the standard coagulation model, planets grow in a circumstellar disk through repeated collisions and mergers of smaller objects (Safronov 1969). First, roughly $\mathrm{km}$ size planetesimals form rapidly, whether by coagulation (e.g. Weidenschilling 2000) or direct collapse (e.g. Goldreich \& Ward 1973). Little knowledge of which process dominates means the size distribution of the first planetesimals is poorly constrained. Planetesimals initially grow through a rapid phase of "runaway" growth (Kokubo \& Ida 1996). During the period of "oligarchic" growth that follows (Kokubo \& Ida 1998), protoplanetary growth rates depend on the surface density of planetesimals $\sigma_{\mathrm{s}}$, the local orbital frequency $\Omega$, the gravitational reach of the growing protoplanet, and the random velocities of the smaller planetesimals (Inaba et al. 2001)

$$
\dot{M}_{\mathrm{pl}} \propto \sigma_{\mathrm{s}} r_{\mathrm{H}}^{2} \Omega P_{\mathrm{col}}(\tilde{e}, \tilde{i}) .
$$

Here $r_{\mathrm{H}}=a\left(M_{\mathrm{pl}} / 3 M_{\star}\right)^{1 / 3}$ is the Hill radius, and $a$ is semi-major axis. The eccentricity $\tilde{e}$ and inclination $\tilde{i}$ are in units of the growing protoplanets Hill radius (i.e. $\left.\tilde{e}=e / r_{\mathrm{H}}\right)$. The collision probability $P_{\text {col }}$ largely determines how growth proceeds: growth is fastest when planetesimals are small enough $(\lesssim 1 \mathrm{~km})$ to be damped by gas drag (e.g. Rafikov 2004). In this "shear dominated" regime when $\tilde{e}$ and $\tilde{i}$ are $\lesssim 1$, growth depends on Keplerian shear in the disk, rather than objects random velocities. Growth slows strongly with increasing radial distance, because $\Omega \propto a^{-3 / 2}$ and $\sigma_{\mathrm{s}} \propto a^{-\delta}$, where $\delta \sim 1-$ 1.5 .

Eventually, protoplanets accrete most of the nearby material and reach the "isolation" mass (Lissauer 1987)

$$
M_{\text {iso }}=\frac{\left(4 \pi B \sigma_{\mathrm{s}} a^{2}\right)^{3 / 2}}{\left(3 M_{\star}\right)^{1 / 2}} .
$$

Numerical simulations indicate that isolated oligarchs are spaced at $2 B R_{\mathrm{H}} \sim 8 R_{\mathrm{H}}$ intervals (e.g. Kokubo \& Ida 1998). In the terrestrial region around the Sun, the isolation mass is $\sim 0.1 M_{\oplus}$, and the timescale for Earth formation by the chaotic growth that follows is $\sim 10-100 \mathrm{Myr}$ (e.g. Kenyon \& Bromley 2006). 
Further out in the disk, larger isolation masses allow formation of gas giant planets. The critical core mass for gas accretion depends on opacity and planetesimal accretion rates, but is $\gtrsim 10 M_{\oplus}$ (e.g. Ikoma et al. 2000; Rafikov 2006). This mass is reached more easily further out in the disk because $M_{\text {iso }}$ increases with $a$. However, growth slows rapidly with increasing radial distance; thus, there is an optimum region where cores are massive enough to accrete gas and to form giant planets before the gas disk is dissipated (Kennedy \& Kenyon 2008). This region is sufficiently far from the star that in situ formation of "hot-Jupiters" is unlikely, thus motivating theories of migration and scattering.

\subsection{Migration}

Type I migration is a potential barrier to the formation of both terrestrial and giant planets (Goldreich \& Tremaine 1980; Ward 1997; Tanaka et al. 2002; Papaloizou et al. 2007). When protoplanets reach near an Earth mass, the excitation of spiral density waves in the gaseous disk causes planets to experience a torque, and migrate inward. The timescale for a planet to spiral into the central star is (Tanaka et al. 2002)

$$
\tau_{\text {mig }}=(2.7+1.1 \delta)^{-1} \frac{\left(M_{\star} M_{\odot}\right)^{2}}{M_{\mathrm{pl}} \sigma_{\text {gas }} a^{2}} \frac{h^{2}}{\Omega},
$$

where $h \approx 0.05$ is the disk aspect ratio, and the stellar mass $M_{\star}$ is in units of Solar masses. For a planet of mass $M_{\mathrm{pl}}=1 M_{\oplus}$ in a disk with $\sigma_{\text {gas }}=1700 \mathrm{~g} \mathrm{~cm}^{-2}$ at $1 \mathrm{AU}$ around a Solar-mass star, $\tau_{\mathrm{mig}}=1.6 \times 10^{5} \mathrm{yr}$. Because this timescale is shorter than the $\sim 3$ Myr disk lifetime (Haisch et al. 2001), and comparable with growth timescales, type I migration theory conflicts with terrestrial and giant planet formation in the Solar System (but see Chambers 2006b).

Recent work suggests a reduced migration efficiency can resolve this problem (Ida \& Lin 2008). This "offset" applies to planets $\lesssim 15 M_{\oplus}$ (D'Angelo et al. 2002, 2003) and arises from corotation torques by coorbital material (Masset et al. 2006a). Other ways of reducing (and even reversing) type I migration rates include turbulence arising from the magnetorotational instability (e.g. Nelson \& Papaloizou 2004), and eccentricity driven by planet-planet interactions (Papaloizou \& Larwood 2000).

If planets do not fall onto the central star, migration is a possible mechanism for producing planets on shortperiod orbits (Lin et al. 1996; Brunini \& Cionco 2005; Terquem \& Papaloizou 2007).

\subsection{Scattering}

Planet-planet scattering can also produce planets with short-period, or low periastron $(q)$ orbits. Originally proposed to explain hot-Jupiters (Rasio \& Ford 1996), this scenario has not been applied to low-mass planets.

Scattering favours giant planets on short-period orbits. When a gas giant scatters into a low periastron orbit, tidal interaction with the star can circularise the orbit on reasonable timescales, with $a \sim 2 q$ (Rasio \& Ford 1996). For lower mass planets, long circularisation timescales make circular orbits unlikely (Raymond et al. 2007). However, if the initial scattering region is suf- ficiently close, as for low-mass stars, detection of lowperiastron eccentric planets is possible.

We now consider two different scenarios that form short-period and/or transiting low-mass planets that begin growth near the snow line, across a range of stellar masses. When the snow line enhancement is small, many planets migrate toward close orbits. This scenario has already been studied for Solar-mass stars by Terquem \& Papaloizou (2007). Here, we instead consider cold finger type disks, where a few planets forming near the snow line dominate others forming elsewhere in the disk. We first consider a scattering scenario resulting from in situ growth, and then a migration scenario. We defer discussion of subsequent planetary evolution in final orbits to $\S 5$.

\section{SCATTERING}

Planet-planet scattering is a likely outcome of oligarchic growth. In migration scenarios, protoplanets interact strongly with the gas disk, and they migrate to close-in orbits. However, if the gas disk disperses before planets have time to migrate, or if migration results in no net inward movement, planets form in situ. During oligarchic growth, protoplanets grow on orbits near the limits of dynamical stability, with damping provided by small bodies (e.g. Stewart \& Wetherill 1988; Kokubo \& Ida 1998). At later stages near isolation, their orbits can become unstable as remaining small bodies are accreted (Goldreich et al. 2004; Kenyon \& Bromley 2006).

When planets start interacting dynamically, the boundary in semi-major axis between stable and unstable configurations is very sharp. Thus, two planets with orbits that become too close experience the sudden onset of a dynamical instability caused by close encounters (Gladman 1993).

In previous studies of giant planet scattering, planets begin at $\sim \mathrm{AU}$ distances from the central star, with spacings just inside the stability limit. After many interactions, one planet sometimes attains a highly eccentric orbit with a small periastron distance (e.g. Rasio \& Ford 1996; Ford \& Rasio 2007). Tidal interaction with the central star then circularises the orbit with $a \sim 2 q$.

While tidal forces can circularise gas giant orbits, the timescales for $1-10 M_{\oplus}$ planets on highly eccentric orbits are long ( $\gtrsim$ Gyr, Raymond et al. 2007). Although these planets maintain eccentric long-period orbits, transits are possible in favourable circumstances. Because planets form at shorter orbital periods around low-mass stars, these provide the best opportunity for transit observations.

Cold finger disks provide an ideal environment for oligarchic growth followed by planet-planet scattering. The width of the cold finger region allows several protoplanets to form (Ciesla \& Cuzzi 2006). Once protoplanets reach isolation, further chaotic growth may occur if their escape velocity $v_{\text {esc }}$ is less than the local Keplerian velocity $v_{\mathrm{K}}\left(\mathcal{R} \equiv v_{\mathrm{esc}} / v_{\mathrm{K}}\right.$, Goldreich et al. 2004). In the terrestrial region of Solar-type stars, $\mathcal{R} \sim 1 / 4$. For gas giants, $\mathcal{R} \gg 1$. For $M_{\mathrm{pl}}=10 M_{\oplus}$ with density $\rho=4.5 \mathrm{~g} \mathrm{~cm}^{-2}$, $\mathcal{R} \approx 1.3$ outside the snow line. Thus, $\sim 10 M_{\oplus}$ protoplanets present an approximate division between coalescence and scattering/ejection, and an order of magnitude estimate of the maximum planet mass. This mass is similar 
TABLE 1

SCATTERING SimUlation OUTCOMES, AND FRACTION With LOW PERIASTRA.

\begin{tabular}{ccccc}
\hline \hline$M_{\star}\left(M_{\odot}\right)$ & collisions & ejections & survival & $q<0.1 \mathrm{AU}$ \\
\hline 0.25 & $95 \%$ & $2.5 \%$ & $2.5 \%$ & $4 \%$ \\
0.5 & $95 \%$ & $0 \%$ & $5 \%$ & $0 \%$ \\
1 & $94 \%$ & $0 \%$ & $6 \%$ & $0 \%$ \\
2 & $84 \%$ & $0 \%$ & $16 \%$ & $0 \%$ \\
\hline
\end{tabular}

to the minimum needed for gas accretion, so scattering of super-Earths to close-in orbits appears difficult.

For less massive stars, scattering to low periastron orbits is easier. At fixed $a$, smaller $v_{\mathrm{K}}$ leads to larger $\mathcal{R}$ and a greater chance of scattering. However, the snow line also moves inward as stellar mass decreases $\left(a_{\text {snow }} \propto\right.$ $M_{\star}^{1-2}$, e.g. Ida \& Lin 2008; Kennedy \& Kenyon 2008), so scattering remains difficult. For $a_{\text {snow }} \propto M_{\star}$, $v_{K}\left(a_{\text {snow }}\right)$ is constant for different stellar masses. However, for a fixed time period, a greater number of conjunctions for low-mass stars allows dynamical evolution to greater eccentricities.

\subsection{Scattering Simulations}

To measure the likelihood of planet-planet scattering, we performed simulations over a range of stellar masses with the MERCURY integrator (Chambers 1999). We initialised integrations with two $10 M_{\oplus}$ planets spaced near the Hill stability criterion to ensure close encounters (Gladman 1993). This planet mass is an approximate maximum mass before cores accrete gas to become gas giants, and thus offers the best chance for scattering over coalescence. To represent a linearly stellar mass dependent snow line, the inner planet was placed at $a_{\text {in }}=$ $3 M_{\star}$ AU. The outer planet begins at a random $a$ in the range 0.9-1 $a_{\text {in }}\left(1+\Delta_{\text {crit }}\right)$, where $\Delta_{\text {crit }}=3\left(M_{\mathrm{pl}} / M_{\star}\right)^{1 / 3}$ (Gladman 1993; Ford \& Rasio 2007). Both planets begin in circular orbits with random inclinations less than $3^{\circ}$; the remaining orbital elements are chosen randomly. Simulations were run with a 5 day timestep for $1 \mathrm{Gyr}$ around stars of $0.25,0.5,1$, and $2 M_{\odot}$, or halted earlier in the case of collisions (we assume perfect mergers) or ejections. A total of 520 simulations were run, 130 for each stellar mass.

\subsection{Scattering Results}

The simulations result in three different outcomes: collisions, ejections, or survival of both planets for $1 \mathrm{Gyr}$. No planets achieved periastra low enough to fall onto the central star. Most (> 85\%) simulations resulted in collisions (Table 1). Some systems survived for the full simulation. The only ejections were for $0.25 M_{\odot}$.

With so few systems remaining after $1 \mathrm{Gyr}$, we use the smallest periastron distance reached in each simulation to characterise the success of planet-planet scattering, shown in Figure 1. As expected, the closer snow line distance for the $0.25 M_{\odot}$ allows smaller periastra after scattering.

For simulations of $0.25 M_{\odot}$ stars, $5 / 130$ (4\%) planets reach periastra less than $0.1 \mathrm{AU}$. A shorter orbital period allows many more conjunctions. Thus, systems evolve further than for more massive stars. For the three ejections, the lowest periastra were reached just before

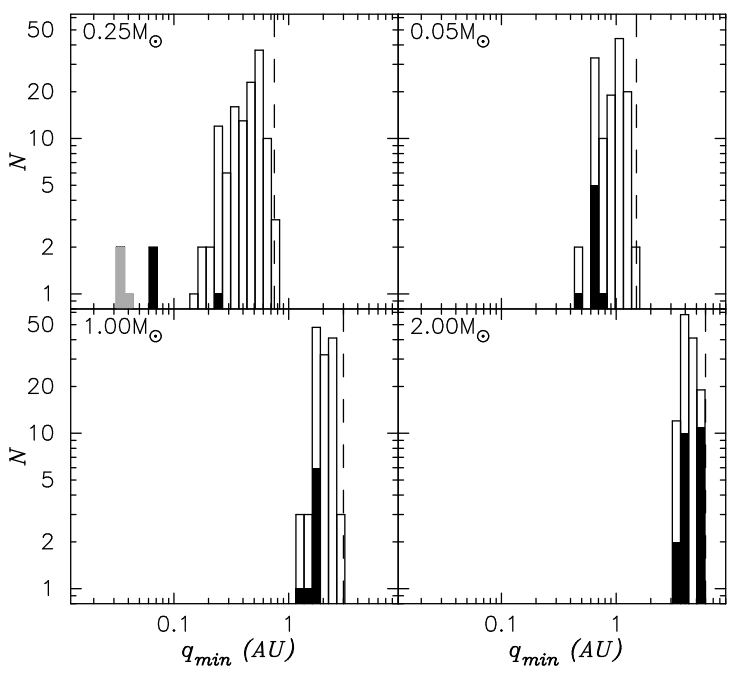

FIG. 1. - Smallest periastra of scattering simulations for all nonstable orbits. Black filled bars: simulations in which both planets survived until 1 Gyr. Grey bars: ejections (orbits going beyond 1000 AU). Unfilled bars: collisions/mergers. The dashed line shows the starting $a$ (and $q$ ) of the inner planets (circular) orbit. Planets are scattered to lower periastra for low-mass stars because of more conjunctions, and starting closer to the central star.

a series of close encounters, which resulted in the ejection. For the three surviving systems, the lowest periastra were reached near the end of the integrations. These orbits have eccentricities $\approx 0.5$, and semi-major axes $\approx 0.5 \mathrm{AU}$, corresponding to an orbital period of around 260 days. Circularisation times for these planets are 10 Gyr (Goldreich \& Soter 1966; Raymond et al. 2007).

Therefore, in the case of $10 M_{\oplus}$ planet-planet scattering, only the lowest mass stars have planets with periastra close enough for transiting orbits. However, long circularisation timescales mean these planets will likely remain on highly eccentric orbits, with periods long enough to make radial velocity and transit detections difficult.

\section{MIGRATION}

We now turn to a migration scenario. In this picture, the largest protoplanets form near the snow line by oligarchic growth. Once they reach masses of $\sim 1-10 M_{\oplus}$, these icy protoplanets migrate towards the central star. In a cold finger disk, migrating icy objects dominate smaller interior rocky protoplanets. Long chaotic growth timescales mean that as the icy object migrates through the terrestrial region, interior rocky objects are not accreted. They are instead scattered outward or shepherded inward. Shepherding - where interior objects are captured into mean-motion resonances - results in rocky protoplanets being pushed inward ahead of the migrating icy planet. These smaller objects merge to form large rocky planets, which are eventually accreted by the larger icy migrator or survive on an interior orbit. We assume that all objects halt their migration when they reach the inner edge of the gas disk, at $\sim 10$ stellar radii.

Our goal is to calculate the growth and migration of individual protoplanets in this scenario. If several protoplanets migrate, whether they do so as a set in resonant orbits (Terquem \& Papaloizou 2007), or successively (e.g. Daisaka et al. 2006; Ida \& Lin 2008), the resulting trends are similar in our picture. The main dif- 
ference between outcomes is the number of icy planets on short-period orbits. The trends our models predict depend largely on disk and planet properties, not multiplicity.

When an icy protoplanet migrates, it only interacts dynamically with interior objects. Because collision cross sections are essentially geometric, the timescale for growth is much longer than the migration timescale. For example, the migration timescale for an Earth-mass planet at $1 \mathrm{AU}$ (several $10^{5} \mathrm{yr}$, or several $10^{6} \mathrm{yr}$ if migration is less efficient) is much smaller than the chaotic growth timescale $\left(\tau_{\text {chaotic }} \sim \rho R_{\mathrm{pl}} / \sigma_{s} \Omega \sim 10^{8} \mathrm{yr}\right.$, where $R_{\mathrm{pl}}$ is the planet radius, Goldreich et al. 2004). Thus, the migrating protoplanet does not accrete terrestrial protoplanets; outward scattering or inward shepherding are the most likely outcomes.

The evolution of interior protoplanets depends on their random velocities. Chaotically growing objects with high eccentricities are scattered outward by the migrating protoplanet. These may interact with another migrating protoplanet or resume chaotic growth. If interior objects have finished chaotic growth and are damped by the gas disk onto more circular orbits, shepherding by capture onto resonant orbits is possible. Shepherded objects merge and form rocky planets as their orbits are pushed together by the migrating icy protoplanet. Shepherding by giant planets undergoing type II migration has been proposed as a way to form super Earth-mass planets (Zhou et al. 2005). However, studies have yet to consider shepherding by super-Earths undergoing type I migration.

While some planets are stranded at intermediate radii as the gas disk dissipates, most planets that begin to migrate reach the inner disk edge, and might fall onto the star. Because the torque on the migrating planet changes when the disk gas surface density profile varies rapidly, as happens at the inner disk edge, this fate may be avoided (Tanaka et al. 2002). Here, corotation torques affect migration, and allow for planets to cease migration before reaching the stellar surface (Masset et al. 2006b). In our migration simulations, we therefore assume migration stops inside the inner disk edge (Terquem \& Papaloizou 2007).

In the rest of this section, we consider three models that explore different aspects of the migration scenario, and observable trends that probe stellar and disk properties. We consider the simplest scenario - when growth is so fast that planets reach isolation before migration begins - with an analytic model in $\S 4.1$. As the planetesimal size increases, growth slows; the timescale becomes comparable to that for migration. The assumption made in the analytic model no longer applies, and we use a semi-analytic model to study concurrent growth and migration in $\S 4.2$. Finally, we use $n$-body simulations in $\S 4.3$ to show the shepherding effects migrating super-Earths have on terrestrial material.

\subsection{An Analytic Approach}

If we assume that protoplanets reach isolation before migration starts, then we can create a simple analytical model for our migration scenario. At isolation, protoplanets have a known migration timescale, which is shorter than the disk lifetime if they are to reach the central star. To remain in the super-Earth mass regime, the mass of a protoplanet is smaller than the critical core mass for gas accretion. Because the isolation mass changes with surface density - and thus with disk massonly a certain range of disk masses satisfy these conditions for fixed stellar mass. To consider a range of different stars, we also consider how the snow line where these migrating planets form - changes with stellar mass. The range of disk masses that satisfy the conditions changes with stellar mass, resulting in potentially observable trends that test migration models.

To begin, we adopt a relation for the surface density of solid material in the disk. In the standard MMSN model,

$$
\sigma_{\mathrm{s}}=\sigma_{0} f_{\mathrm{ice}} a_{\mathrm{AU}}^{-\delta},
$$

where $\sigma_{0}=8 \mathrm{~g} \mathrm{~cm}^{-2}, \delta=1-1.5$, and $a_{\mathrm{AU}}$ is $a$ in units of AU. The factor $f_{\text {ice }} \sim 2-3$ is the enhancement from ice condensation beyond the snow line. This disk has a mass $\sim 0.01 M_{\odot}$.

To generalise this relation, we add terms to account for differences in disk mass and metallicity around stars with a range of masses. Disks around young stars have a large dispersion in mass (Natta et al. 2000; Andrews \& Williams 2005, 2007). Setting the disk mass $M_{\text {disk }} \propto \eta M_{\star}^{\beta}$ allows us to treat the observed trends with stellar mass- $M_{\text {disk }} \propto M_{\star}^{\beta}$, with $\beta \approx 1$-and a range $(\eta)$ of disk masses at fixed stellar mass. Adopting a factor $\mathcal{M} \propto 10^{[\mathrm{Fe} / \mathrm{H}]}$ for the metallicity of the stars and the disk yields

$$
\sigma_{\mathrm{s}}=\sigma_{0} \eta f_{\text {ice }} \mathcal{M} M_{\star}^{\beta} a_{\mathrm{AU}}^{-\delta} .
$$

For simplicity, we combine $f_{\text {ice }}$ and $\mathcal{M}$ into a single factor $\Delta=f_{\text {ice }} \mathcal{M}$, which quantifies the enhancement of solid material relative to gas where these planets form. For a cold finger disk, we use $f_{\text {ice }}=10$. Thus, for typical ranges in $\mathcal{M}(\sim 1 / 3-3)$ and $f_{\text {ice }}(2-10)$, the plausible range of $\Delta$ is $0.6-30$. We concentrate on higher $\Delta$, because these are cold finger disks.

For the surface density of the gas disk, we set $\sigma_{g}=$ $100 \sigma_{s} / \Delta$. Thus, the gas mass depends on $\eta$ and $\delta$, and is independent of metallicity and the enhancement in ices at the snow line. We adopt $\delta=3 / 2$.

How the snow line varies with stellar mass is uncertain. The existence of gas giant planets suggests that the stages of planet formation up to isolation occur while the gas disk is still present. During these stages the snow line distance is set by viscous accretion of the gas disk. If the accretion rate onto the star is $\dot{M} \propto M_{\star}^{1-2}$, then $a_{\text {snow }} \propto M_{\star}^{6 / 9-8 / 9}$ (Kennedy \& Kenyon 2008). Later, when the star has reached the main-sequence and the gas disk has been dissipated, the main-sequence luminosity is more important and $a_{\text {snow }} \propto M_{\star}^{2}$ (Ida \& Lin 2005). Because we model oligarchic growth, and $\dot{M} \propto M_{\star}^{2}$ (Muzerolle et al. 2005), we adopt the snow line distance $a_{\text {snow }}=2.7 M_{\star} \mathrm{AU}$. Variation of the snow line with time and stellar mass is a key component of planet formation models that consider a range of spectral types (Kennedy \& Kenyon 2008).

Substituting our adopted surface density into the isolation mass yields

$$
M_{\text {iso }} \propto \frac{\left(\sigma_{\mathrm{s}} a^{2}\right)^{3 / 2}}{\left(M_{\star}\right)^{1 / 2}}=\frac{\left(\eta f_{\text {ice }} \mathcal{M} M_{\star} a^{1 / 2}\right)^{3 / 2}}{M_{\star}^{1 / 2}} .
$$


The isolation mass increases with any parameter that increases the surface density. The increasing disk mass with stellar mass $\left(M_{\star}\right.$ in numerator) is stronger than the decreasing Hill radius ( $M_{\star}$ in denominator). Thus, at fixed $a$ the isolation mass increases with stellar mass. For our scenario, we are interested in planets that form at the snow line, so the changing snow line distance $(a=$ $a_{\text {snow }} \propto M_{\star}$ ) makes the stellar mass dependence stronger.

Substituting $a=2.7 M_{\star} \mathrm{AU}$, equation (6) yields the isolation mass at the snow line for a range of stellar and disk masses, and metallicities and snow line enhancements

$$
M_{\text {iso }}=0.12(\Delta \eta)^{3 / 2} M_{\star}^{7 / 4} M_{\oplus} .
$$

Applying the same approach to type I migration yields

$$
\tau_{\text {mig }} \propto \frac{M_{\star}^{2} h^{2} f_{\text {mig }}}{a^{2} M_{\mathrm{pl}} \Omega \sigma_{\text {gas }}}=\frac{M_{\star}^{1 / 2} h^{2} f_{\text {mig }} a}{M_{\mathrm{pl}} \eta},
$$

where the offset $f_{\text {mig }}$ allows us to consider reduced migration rates. At fixed $a$, migration takes longer as stellar mass increases, and speeds up as planet mass increases. If planet masses vary less strongly with radial distance than $M_{\mathrm{pl}} \propto a$, then the migration timescale increases outward, and planets cannot catch up to interior ones. Even with isolated objects $\left(M_{\text {iso }} \propto a^{3 / 2}\right)$, planets may not catch up to interior ones due to the strong slowing of growth with semi-major axis. At the snow line distance, migration slows even more strongly with increasing stellar mass due to lower gas density and slower orbital periods at larger radii.

Again substituting $a=2.7 M_{\star} \mathrm{AU}$, the timescale to migrate from the snow line to the star is

$$
\tau_{\mathrm{mig}}=9.1 \times 10^{5} \frac{f_{\mathrm{mig}} M_{\star}^{3 / 2}}{M_{\mathrm{pl}} \eta} \mathrm{yr},
$$

where we have set $h=0.05$ (e.g. Papaloizou et al. 2007). At fixed $M_{\star}$, massive planets in massive disks migrate to the inner disk edge fastest. The migration timescale increases with $M_{\star}$ because the snow line is further away.

If the migration time is shorter than the disk lifetime (i.e. $\tau_{\text {mig }} \lesssim \tau_{\text {disk }} \sim 1 \mathrm{Myr}$ ), then protoplanets reach short-period orbits. This inequality leads to

$$
M_{\mathrm{pl}}>\frac{0.91 f_{\mathrm{mig}} M_{\star}^{3 / 2}}{\eta} M_{\oplus} .
$$

This result yields the minimum mass for a planet to migrate to a close orbit. Substituting the isolation mass (eq. 7) for $M_{\mathrm{pl}}$ and solving for $\eta$ gives a lower relative disk mass limit of

$$
\eta>\eta_{\text {low }}=\frac{2.2 f_{\mathrm{mig}}^{2 / 5}}{M_{\star}^{1 / 10} \Delta^{3 / 5}} .
$$

Disks more massive than this $\eta$ form protoplanets massive enough to migrate to short-period orbits before the gas disk dissipates. Planets in slightly less massive disks still migrate, but are stranded at intermediate radii as the disk disperses.

The critical $\sim 10 M_{\oplus}$ core mass for gas accretion provides an upper limit for the protoplanet mass. Solving
$M_{\text {iso }}<10 M_{\oplus}$ for $\eta$ yields

$$
\eta<\eta_{\mathrm{hi}}=\left\{\begin{array}{ll}
\frac{18.6}{M_{\star}^{7 / 6} \Delta} & \frac{18.6}{M_{\star}^{7 / 6} \Delta}<30 \\
30 & \frac{18.6}{M_{\star}^{7 / 6} \Delta} \geq 30
\end{array},\right.
$$

where the additional constraint of a reasonable disk mass sets $\eta \lesssim 30\left(M_{\text {disk }} \lesssim 0.3 M_{\star}\right)$ as an upper limit (e.g. Ida \& Lin 2005). Because we assume growth is fast, planetesimal accretion drops significantly at later stages. The core mass for gas accretion is then somewhat smaller (Ikoma et al. 2000; Rafikov 2006).

The two limits on disk mass yield a simple relation between the stellar mass, migration offset, and enhancement factor. Equating $\eta_{\text {low }}$ and $\eta_{\text {hi }}$,

$$
M_{\star, \max }=\frac{7.3}{\left(f_{\operatorname{mig}} \Delta\right)^{3 / 8}}
$$

in units of Solar masses. ${ }^{1}$ This equation has a simple physical interpretation. For massive $\operatorname{stars}\left(M_{\star}>\right.$ $M_{\star, \max }$ ), the only protoplanets massive enough to migrate to the central star before the gas disk disperses are above the critical core mass for gas accretion. These planets therefore become gas giants, rather than forming hot super-Earths. For lower stellar masses, the closer snow line distance allows planets smaller than the critical core mass to migrate to the host star. Thus, $M_{\star}$,max is the maximum stellar mass for hot super-Earths produced by type I migration.

Making an estimate of $M_{\star, \max }$ requires an assumed $f_{\text {mig }}$ and $\Delta$. For Solar metallicity $\mathcal{M}=1$, and a cold finger enhancement $f_{\text {ice }}=10-20, \Delta=10-20$. For a migration offset $f_{\text {mig }}=10, M_{\star, \max } \sim 1 M_{\odot}$. Transit and radial velocity surveys routinely probe these stellar masses. Independent of the disk mass distribution, this result is therefore a simple testable prediction of hot super-Earth formation by type I migration.

Figure 2 shows the range of planet masses that reach short-period orbits for a range of stellar masses. For the analytic model (thick grey lines) the upper limit is constant at $10 M_{\oplus}$. The lower limit decreases as stellar mass and snow line distance decrease. The expected range of planet masses decreases with increasing stellar mass, while the average mass increases to $10 M_{\oplus}$, where the lines meet at $M_{\star, \max }=1.3 M_{\odot}$.

In addition to this maximum stellar mass, we can derive the probability of forming hot super-Earths around stars with $M_{\star}<M_{\star \text {,max }}$. This estimate requires an adopted distribution of $\eta$ (i.e. disk masses). If relative disk masses $\left(M_{\text {disk }} / M_{\star}\right)$ are distributed as a power law with index $\sim-1.75$ (Andrews \& Williams 2005), the (relative) probability of forming a close-in planet as a function of stellar mass for a given $\Delta$ is

$$
P_{\mathrm{p}}\left(M_{\star}, \Delta\right) \propto \int_{\eta_{\mathrm{lo}}}^{\eta_{\mathrm{hi}}} \eta^{-1.75} d \eta
$$

Alternatively, disk masses may be distributed around some "typical" relative disk mass (e.g. Ida \& Lin 2005)

$$
P_{\mathrm{g}}\left(M_{\star}, \Delta\right) \propto \int_{\eta_{\mathrm{lo}}}^{\eta_{\mathrm{hi}}} \exp \left(-\frac{(\log (\eta)-\mu)^{2}}{2 s^{2}}\right) d \eta
$$

1 Equating (11) and (12) has two solutions for $M_{\star}$ because of the upper limit of 30 . The other solution is at $M_{\star}$ far too small to be interesting. 


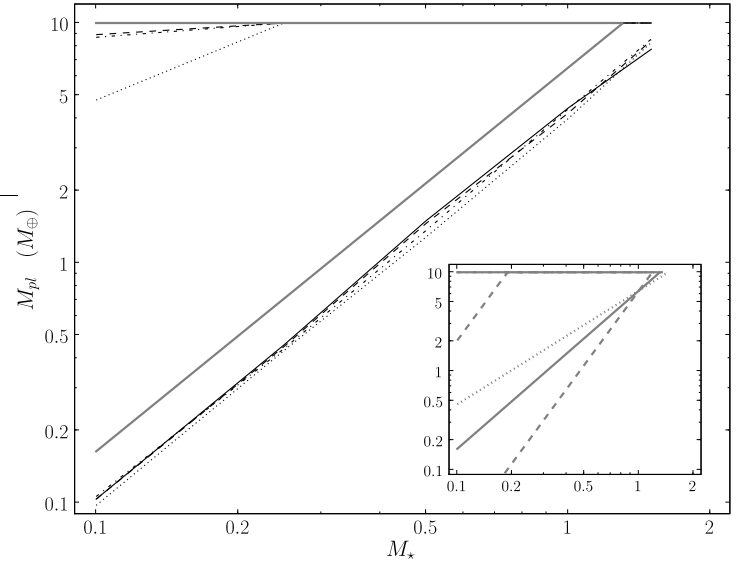

FIG. 2.- The range of planet masses that reach close-in orbits as a function of stellar mass. The thicker grey line line shows results from the analytic model using equations (7), (11), and (12). Other lines show upper and lower mass limits for a range of planetesimal sizes from the semi-analytic model (see $\S 4.2$ ) for $\Delta=10$ : $r=10 \mathrm{~m}$ (plain), $100 \mathrm{~m}$ (dashed), $1 \mathrm{~km}$ (dot-dashed), and $10 \mathrm{~km}$ (dotted). The range of planet masses reaching short-period orbits decreases with increasing stellar mass because the snow line distance is greater. The inset panel (same axes) shows how different snow line relations affect the model (using $a_{\text {snow }}=2.7 M_{\star}^{\alpha} \mathrm{AU}$ ). Lines are for $\alpha=1 / 2$ (dotted), 1 (plain), and 2 (dashed) A more strongly varying snow line distance $(\alpha=2)$ yields smaller $M_{\text {iso }}$ (due to smaller $R_{\mathrm{H}}$ ) at much closer snow line distances as stellar mass decreases.

where we choose the standard deviation $s=1$. This distribution is plausible because opacities may underestimate disk masses by as much as an order of magnitude, due to mass locked up in boulder size objects (Andrews \& Williams 2007). Therefore mm observations see disks not only with a range of masses, but in a range of evolutionary states. Unlike the case for giant planets, there is no observational anchor point, so we present these results as relative probabilities.

For a range of $\Delta$, the left panel of Figure 3 shows the probability distribution for the power law disk mass distribution with $f_{\mathrm{mig}}=10$. Results are similar for $f_{\text {mig }}=1$, with the main difference that $M_{\star, \max }$ is higher (eq. 13). Higher $\Delta$ are most relevant here because low values describe MMSN disks, which result in many similar-mass migrating planets originating from a wide range of radii. The point where lines break and decrease toward lower stellar masses is caused by the maximum disk mass condition $\eta<30$. In these cases the maximum short-period planet mass is not set by gas accretion, and is $<10 M_{\oplus}$. This limit applies when $\Delta \lesssim 5$ for the lower of the stellar masses we consider, so does not apply to cold finger disks with $f_{\text {ice }} \gtrsim 10$ unless they have metallicity $\mathcal{M} \lesssim 0.5$

At the lowest stellar masses, there is a clear increase in planet frequency with $\Delta$. With a power-law distribution of disk masses, the most common disks are the least massive; these require large $\Delta$ to allow them to form planets massive enough to migrate (and satisfy condition 11). Near $M_{\star, \max }$, there is an optimum $\Delta$, which is a balance between the likelihood of different disk masses and the $\Delta$ needed to form close-in planets from those disks. At $M_{\star \text { max }}$, the only planet that reaches a shortperiod orbit has $M_{\mathrm{pl}}=10 M_{\oplus}$. Therefore the range of short-period planet masses decreases up to $M_{\star \text { max }}$. The average planet mass increases with stellar mass.

The right panel of Figure 3 shows the probability distribution for the Gaussian distribution with $\mu=1$. The most common disk mass is thus $\sim 0.1 M_{\star}$. As $\Delta$ increases, the probability of forming a short-period planet decreases once the disk mass distribution is not truncated by the condition $\eta<30$. In contrast to the power law distribution, the low-mass disks requiring large $\Delta$ are uncommon. Thus, as $\Delta$ increases, isolation masses are pushed over the gas accretion mass, and the likelihood of forming close-in $\lesssim 10 M_{\oplus}$ planets decreases. While the curves are different from the left panel, the point $M_{\star, \max }$ is the same for a given $\Delta$. With $\mu=0$ (i.e. distributed about $M_{\text {disk }}=0.01 M_{\star}$ ) the probability distribution is qualitatively similar to the power law disk distribution.

In summary, the simple analytical model yields testable predictions for an ensemble of super-Earths that migrate into short-period orbits from the snow line. For reasonable input parameters, we predict a maximum stellar mass $\sim 1 M_{\odot}$ for stars with close-in super-Earths. If circumstellar disks tend to have similar snow line enhancements, this maximum mass decreases with the metallicity of the host star. For a range of stellar masses, the frequency of hot super-Earths depends on the initial distribution of disk masses. For a power-law (Gaussian) distribution of disk masses, the model predicts more (fewer) hot super-Earths around more metal-rich stars.

To give the these trends some context, the first transiting low-mass planet orbits a star with sub-Solar mass and metallicity (GJ436b, Gillon et al. 2007). The current sample of low minimum-mass planets also indicates a flatter metallicity distribution than exists for giant extraSolar planets (Udry et al. 2007). While both disk mass distributions suggest that low stellar mass host is likely, the power law distribution argues against a low metallicity host. The Gaussian disk mass distribution, centered on a relatively high disk mass is consistent with an increasing giant planet frequency with metallicity, and a flatter or decreasing frequency for lower mass planets.

Disks with $\eta>\eta_{\text {hi }}$ form gas giants. Their relative probabilities can thus be calculated by integrating equations (14) and (15) from $\eta_{\mathrm{hi}}$ to 30. However, because $\eta_{\text {low }}$ only weakly depends on $M_{\star}$, giant planet frequency is roughly some constant minus the hot super-Earth frequency (i.e. generally increases with $M_{\star}$ ). This trend is essentially the result arrived at by previous theoretical studies (e.g. Ida \& Lin 2005; Kennedy \& Kenyon 2008), and is at least qualitatively consistent with the observed trend (Johnson et al. 2007).

In constructing the above model we simplified some parameters, and assumed values for others. We now briefly consider model sensitivity to these, and whether observations may constrain them. The most uncertain simplification is how the snow line distance varies with stellar mass. Within our framework, relaxing the distance to $a_{\text {snow }}=2.7 M_{\star}^{\alpha} \mathrm{AU}$ results in changes to Equations (12), (11), and (13) for $\alpha=1 / 2-2$ (Fig. 2 inset). A more strongly varying snow line distance $(\alpha=2)$ yields much closer $a_{\text {snow }}$ and smaller $M_{\text {iso }}$ (due to smaller $R_{\mathrm{H}}$ ) for low mass stars. A more complex snow line model could include how $a_{\text {snow }}$ varies with $M_{\text {disk }}$ at fixed stellar mass, or some time dependence (e.g. Ciesla \& Cuzzi 2006; Kennedy \& Kenyon 2008).

Another uncertain parameter is $\delta$, the disk surface den- 

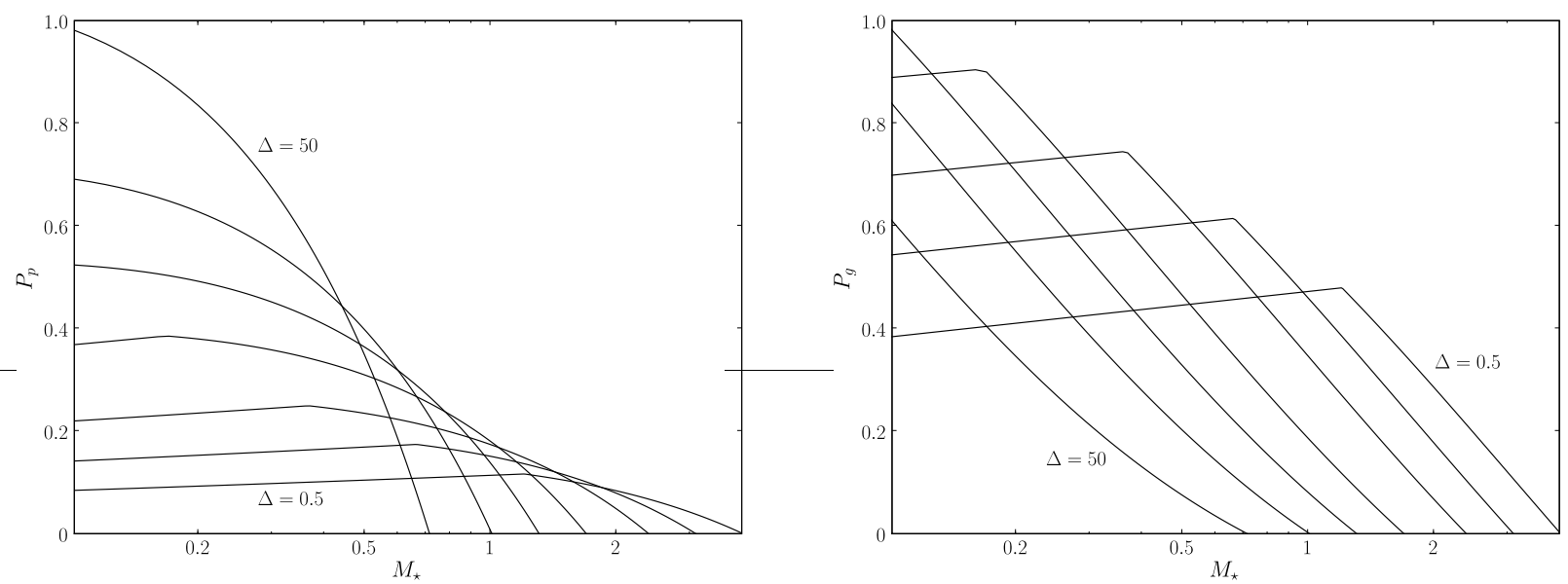

Fig. 3.- Probability distributions for power law (left), and Gaussian with $\mu=1$ (right) disk mass distributions of $\lesssim 10 M_{\oplus}$ planets as a function of stellar mass. For $f_{\mathrm{mig}}=10$, lines are for $\Delta=0.5,1,2,5,10,20$, and 50 from right to left where curves cross the x-axis. Thus $M_{\star, \text { max }}$ decreases with increasing $\Delta$, and is independent of the disk mass distribution). Each plot is arbitrarily normalised to 1 for the most likely $\Delta$ at $M_{\star}=0.1 M_{\odot}$.

sity power-law index. While we used $\delta=3 / 2$, many models also consider $\delta=1$. With $\delta=1$, the main results of Figure 2 are unchanged, with stronger migration accounting for lower mass planets as the snow line distance decreases. It is unlikely observations of shortperiod super-Earths can constrain $\alpha$ or $\delta$ based on Figure 2 , because they affect lower limits to planet masses, which will be hard to detect.

The efficiency of type I migration is also unclear. Our choice of $f_{\mathrm{mig}}=10$ is based on numerical simulations, but may also be probed by future discoveries. The maximum stellar mass $M_{\star, \max }$ is not very sensitive to the snow line distance or disk profile, so for fixed snow line and metallicity enhancements $(\Delta)$, observations probe values for $f_{\text {mig }}$.

Our final major assumption is that planets form rapidly, and reach isolation before migrating. If planetesimals are small and growth is shear dominated, this assumption is generally true. With larger planetesimals however, growth is slower and planets may leave their formation regions while still growing. Planetary growth and migration are then coupled, and must be calculated simultaneously. Recently, Chambers (2006a,b) showed how a semi-analytic model of oligarchic growth can take different planetesimal sizes into account, and estimate their effect on growth rates (see also Thommes et al. 2003; Brunini \& Benvenuto 2008). We now turn to a similar, yet simplified model to estimate the effects of planetesimal size on growth and migration.

\subsection{Semi-Analytic Model}

If planets grow fast enough, the isolation mass sets the range of disk masses that form migrating planets. If planetesimals are large enough, growth is not shear dominated and is slower. Migration then begins before planets reach isolation. To follow this evolution, a model treating concurrent accretion and migration is necessary. Our model tracks damping of planetesimal random velocities by gas drag and stirring by a growing protoplanet. The random velocities set how growth proceeds relative to migration, allowing comparison with the analytic model.

In the model, a single protoplanet of mass $M_{\mathrm{pl}}$ grows on a circular orbit from a planetesimal disk of small bodies of radius $r$. We adopt the accretion rate of Inaba et al. (2001) with the atmosphere enhanced accretion radius of Inaba \& Ikoma (2003). To account for accretion of other nearby protoplanets, the growth rate is increased by $50 \%$ (Chambers 2006b). Planetesimal random velocities are stirred by the growing protoplanet (Ohtsuki et al. 2002) and damped by gas drag (Inaba et al. 2001). The protoplanet accretes and stirs material within an annulus of half-width $4 R_{\mathrm{H}}$, and undergoes type I migration at the rate derived by Tanaka et al. (2002), modified by the offset $f_{\text {mig }}$. We use a ten times less efficient migration rate, motivated by numerical (D'Angelo et al. 2002, 2003; Masset et al. 2006a), and Monte-Carlo simulations (Ida \& Lin 2008). Objects have mass density $\rho=1.5 \mathrm{~g} \mathrm{~cm}^{-3}$ outside the snow line. Simulations are started with planetesimals in an equilibrium between protoplanet stirring and gas drag. Because we consider growth only near the snow line (see below), planetesimals do not undergo radial motions due to gas drag. Planetesimals lost to gas drag can be returned to the growth region by the cold finger mechanism (Cuzzi \& Zahnle 2004). The system is evolved using 4th order Runge-Kutta integration with an adaptive step-size (Press et al. 1992).

As before, we model protoplanets that form just outside the snow line. These are the largest objects that migrate to the central star in a cold finger disk and are largely unaffected by interior objects. However, a migrating protoplanet shepherds material inward as it migrates, and will accrete some terrestrial material. This accretion cannot be treated by the semi-analytic model, so protoplanets cease accretion once they pass inside the snow line in the semi-analytic model. We model shepherding with $n$-body simulations in $\S 4.3$. We vary $\eta$ to form $1-10 M_{\oplus}$ planets and use $\Delta=10$.

Protoplanets begin with masses $1 \times 10^{-4} M_{\oplus}$, at $4 R_{\mathrm{H}}$ outside the snow line. This starting condition allows them to reach isolation if growth is faster than migration. The disk is split into 1000 equally spaced radial bins. However, because accretion inside the snow line is turned off, objects grow from material in $\sim 100$ bins 


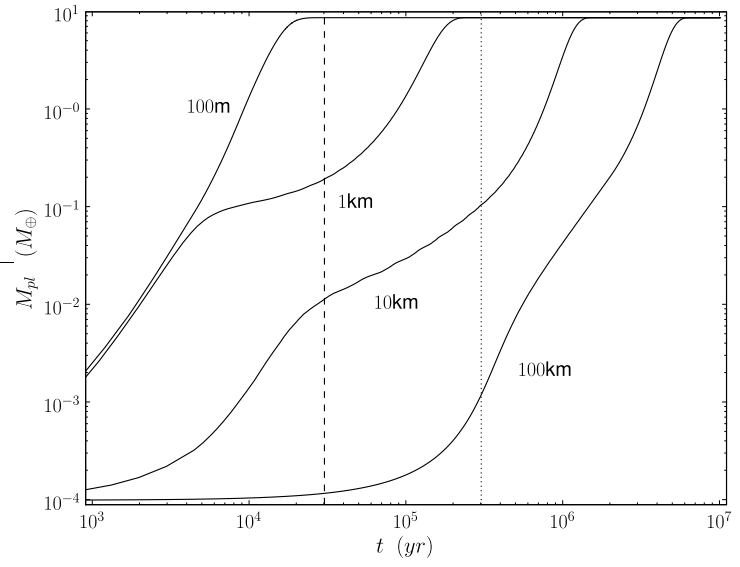

FIG. 4.- Growth to isolation of a protoplanet at $5 \mathrm{AU}$ around a Solar-mass star. Isolation times are in good agreement with Figure 1 of Chambers (2006b). Both models have $\sigma_{0}=10 \mathrm{~g} \mathrm{~cm}^{-2}$ and $\sigma_{\mathrm{g}}=900 \mathrm{~g} \mathrm{~cm}^{-2}$. Our model includes explicit calculation of planetesimal eccentricities and inclinations, which accounts for differences. Lines are for $r=100 \mathrm{~m}, 1 \mathrm{~km}, 10 \mathrm{~km}$, and $100 \mathrm{~km}$ from left to right. The dashed (dotted) lines show the type I migration timescale for $f_{\mathrm{mig}}=1(10)$.

outside the snow line.

The snow line distance and gas disk are as in $§ 4.1$ (eq. 5 and following text), but the surface density of the gas disk decays exponentially with an e-folding time of $1 \mathrm{Myr}$. We place the inner edge of our disk at $0.2 M_{\star} \mathrm{AU}$, though planets that reach a few tenths of an $\mathrm{AU}$ are migrating so rapidly that the exact value matters little.

To test our code, we compare growth at $5 \mathrm{AU}$ with Figure 1 from Chambers (2006b). His figure compares isolation times for different $r$ with the type I migration timescale. The smallest size planetesimals allow protoplanets to reach isolation before migration starts. Growth was simulated at $5 \mathrm{AU}$ around a Solar-mass star, with a solid surface density of $10 \mathrm{~g} \mathrm{~cm}^{2}$ (so $M_{\text {iso }} \approx$ $10 M_{\oplus}$ ), and a gas/solids ratio of 90 . Migration was not included, and the isolation time was simply compared to the analytic estimate of Equation (3).

Figure 4 shows growth in the absence of migration at $5 \mathrm{AU}$ around a Solar-mass star for a range of $r$ with similar initial conditions. The time to reach isolation is fastest for the smallest $r(100 \mathrm{~m})$, because growth is always shear dominated. For $r=1 \mathrm{~km}$, the growing protoplanet excites the small body random velocities. Growth ceases to be shear dominated at several $10^{3} \mathrm{yr}$. For higher $r$, isolation takes even longer, due to the decreasing effectiveness of gas drag on larger planetesimals. Compared with Figure 1 from Chambers (2006b), the time to reach $10 M_{\oplus}$ is in good agreement. Our explicit calculation of eccentricities and inclinations accounts for differences in how growth proceeds (c.f. Fig. 3 of Chambers 2006a).

Models with sufficiently small planetesimals reach isolation before migration. With $f_{\mathrm{mig}}=1, r \lesssim 100 \mathrm{~m}$, and for $f_{\text {mig }}=10, r \lesssim 1 \mathrm{~km}$. Thus, even with a reduced migration rate, protoplanets may still migrate before isolation.

\subsubsection{Semi-Analytic Model Results}

For the range of disk masses $(\eta)$ that forms $1-10 M_{\oplus}$ planets around stars with masses $0.25-2 M_{\odot}$, Figure 5 shows semi-major axis and mass evolution for $r=100 \mathrm{~m}$.
The choice of $1 M_{\oplus}$ is somewhat arbitrary, but represents a rough lower limit for detection. We first describe the Solar case, and then look at differences as the stellar mass, and $r$ change.

For a Solar mass star, growth is not always fast enough for migration to occur before the gas disk is dispersed. For $\eta=2$, the objects Hill radii increase faster than small bodies are stirred; thus growth remains shear dominated $(\tilde{e}, \tilde{i} \lesssim 1)$. The protoplanet successfully migrates to the inner edge of the disk. For lower $\eta$, stirring overcomes damping at several $\times 10^{3} \mathrm{yr}$ and growth slows. Higher $\eta$ results in faster growth of larger objects, which migrate early enough to avoid stalling at intermediate radii. With $\eta=1$, migration is somewhat significant, and the $\sim 3$ Earth mass planet stalls at $\sim 1 \mathrm{AU}$ due to dissipation of the gaseous disk. For $\eta=0.5$, the Earth-mass planet migrates little, and remains beyond the snow line. Final planet masses, and the degree of migration, are set by the initial surface density beyond the snow line.

We turn now to trends across a range of stellar masses. Because of smaller snow line distances, migration is easiest for planets in the $1-10 M_{\oplus}$ range around lower mass stars. Low-mass stars are the most likely to form these planets, because the range of disk masses that form them is much larger. For higher mass stars the more distant snow line makes migration unlikely for all but the most massive planets. Growth is driven out of the shear dominated regime more easily due to lower gas density at greater distances. This result confirms the maximum stellar mass $M_{\star \text { max }}$ described above. As in the analytic model, $M_{\star \text {, max }}$ lies between $1-2 M_{\odot}$ with $\Delta=10$, because no planet with a mass $\lesssim 10 M_{\odot}$ migrates significantly for $2 M_{\odot}$. As stellar mass increases, the relative disk mass required to form $1-10 M_{\oplus}$ planets decreases (eq. 7).

Figure 6 shows how growth changes if planetesimals are larger. Models again have $\Delta=10$, but now the planetesimal radius $r=10 \mathrm{~km}$. For larger planetesimals growth is easily stirred out of the shear dominated regime by the large objects for all stellar masses. The disk masses needed to reach the same range of planet masses are higher, because planets migrate out of the accretion region before they reach isolation. For $0.25 M_{\odot}$ stars, the maximum $\eta=30$ only just forms $10 M_{\oplus}$ planets. Again, $M_{\star, \max }$ lies between 1-2 $M_{\odot}$, indicating that it is largely independent of planetesimal size. Though growth is slower, the results for $r=10 \mathrm{~km}$ are largely the same as $100 \mathrm{~m}$, because the surface density can be increased to account for the slower growth.

Figure 2 also includes results from the semi-analytic model, showing the range of planet masses that reach short-period orbits for a range of stellar masses. Models were run for $\Delta=10$, with $r=10 \mathrm{~m}-10 \mathrm{~km}$ and $M_{\star}=$ $0.1-2 M_{\odot}$. The upper limit decreases at $0.1 M_{\odot}$ due to an upper limit on disk masses. Results from the analytic model are in good agreement. The difference in the lower limit arises because migration is faster at smaller radii, allowing smaller planets to reach the inner disk edge in the semi-analytic model.

In summary, using a more detailed migration model yields results similar to the simple analytic treatment in $\S 4.1$. The inclusion of growth rates due to different planetesimal sizes adds another dimension due to differ- 


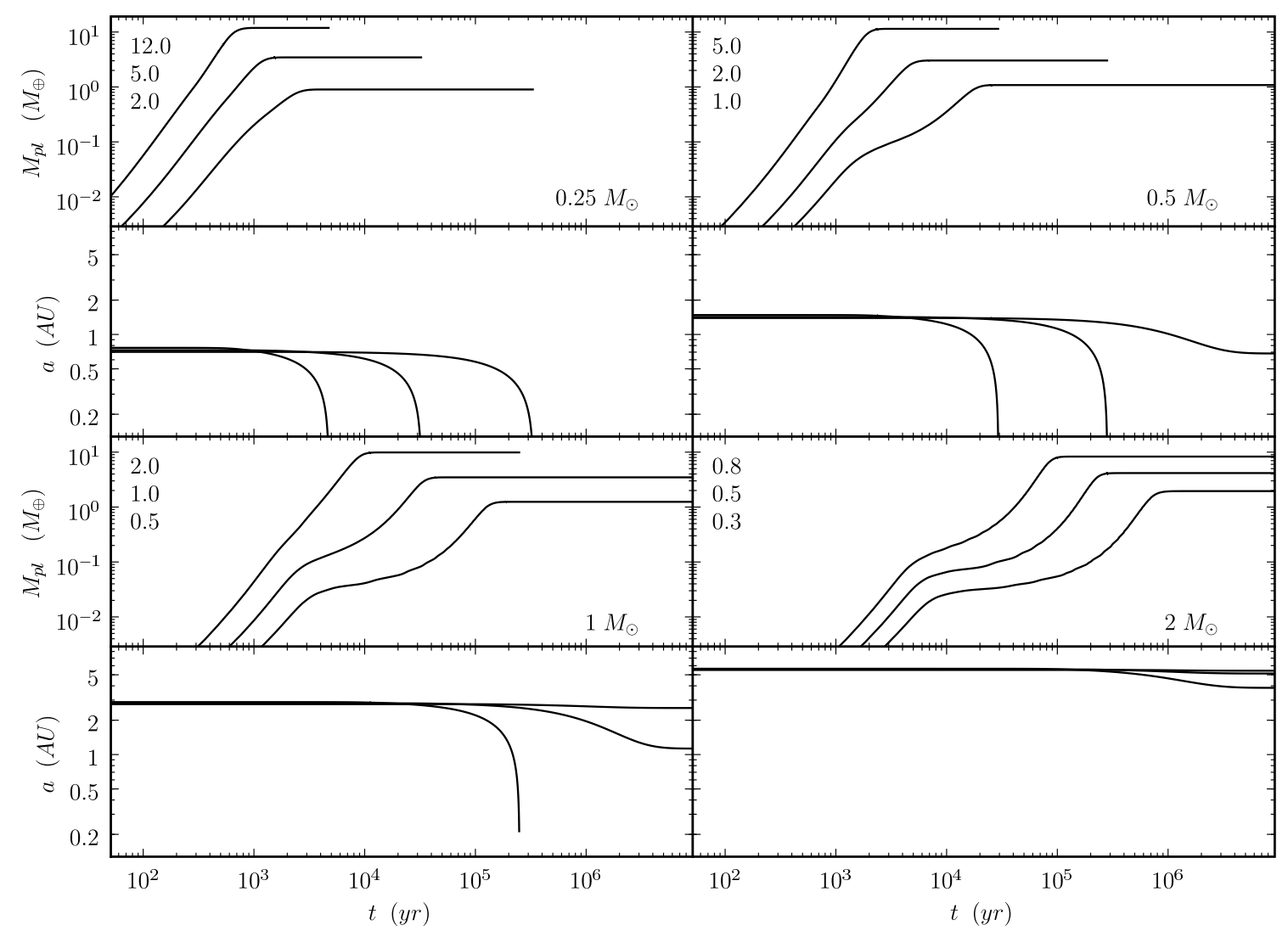

FIG. 5.- Results from the semi-analytic cold finger disk model with $\Delta=10$ and $r=100 \mathrm{~m}$. Each vertical pair of panels shows mass and semi-major axis evolution for several relative disk masses. Higher disk masses ( $\eta$ in legends) form more massive planets that migrate earlier. All $1-10 M_{\oplus}$ planets migrate to the inner disk edge for $0.25 M_{\odot}$, while none do for $2 M_{\odot}$.

ent relative timescales for migration and accretion. The model offers more insight into how growth proceeds, and how the growth rate sets the required disk mass for forming short-period planets.

\subsection{Shepherding}

As a large body migrates inward, it captures interior objects onto mean motion resonances, and shepherds them inward. In the original scenario, a gas giant forms near the snow line, and subsequently migrates inward. As the giant migrates it shepherds interior protoplanets inward, which collide and merge to form super Earthmass planets (Zhou et al. 2005). Here we use $n$-body simulations to study a similar scenario, but with a lowmass planet migrating inwards from the snow line due to type I migration.

To investigate shepherding effects, we used the MERCURY integrator (Chambers 1999), including type I migration and damping forces (Cresswell \& Nelson 2006; Fogg \& Nelson 2007). The migration rate, and eccentricity and inclination damping are reduced by a factor of $f_{\text {mig }}=10$. The inner disk edge is placed at $0.05 \mathrm{AU}$, and inside this point planets cease to interact with the disk (Terquem \& Papaloizou 2007). Simulations are initialised with a number of isolated protoplanets in a disk between $0.1 \mathrm{AU}$ and the snow line at $2.7 M_{\star} \mathrm{AU}$.

Isolation masses are calculated from Equation (7) with the half-spacing $B$ randomly varied between 3.75 and 4.25. We assume Solar metallicity and $f_{\text {ice }}=10$. One protoplanet begins beyond the snow line. This outermost protoplanet is $\approx 30$ times more massive than the one immediately interior to it (eq. 2). Initial eccentricities (inclinations) are randomly distributed between 0 and 0.02 $\left(0.5^{\circ}\right)$, and the remaining orbital elements are randomly distributed. We set the mass of the outermost planet at the middle of the range shown for $M_{\star}=0.25,0.5$, and $1 M_{\odot}$ in Figure $2 ; 2,3.2$, and $6 M_{\oplus}$ respectively. Simulations are run for $10^{8} \mathrm{yr}$ with $\sim 0.3$ day timesteps. Objects are allowed to collide, and are assumed to merge into a single body with no fragmentation. These simulations do not include relativistic effects, or tidal interaction with the star. See Terquem \& Papaloizou (2007) for a more detailed study of migration to small radii, and how these effects affect final system dynamics.

\subsubsection{Shepherding results}

Figure 7 shows the semi-major axis evolution resulting from these simulations. All show similar characteristics. Starting from the inner disk edge, a wave of chaotic growth moves outward (e.g. Chambers 2001; Kenyon \& Bromley 2006), until the number of protoplanets is reduced such that their spacing is stable. This stability is set by a balance between mutual perturbations between protoplanets, and damping by interaction with the gas disk.

When the outermost large protoplanet begins to migrate, it scatters the first objects it encounters into exterior orbits. When the interaction occurs, these objects are still undergoing eccentric chaotic growth, and are less likely to be captured onto resonances and shepherded in- 


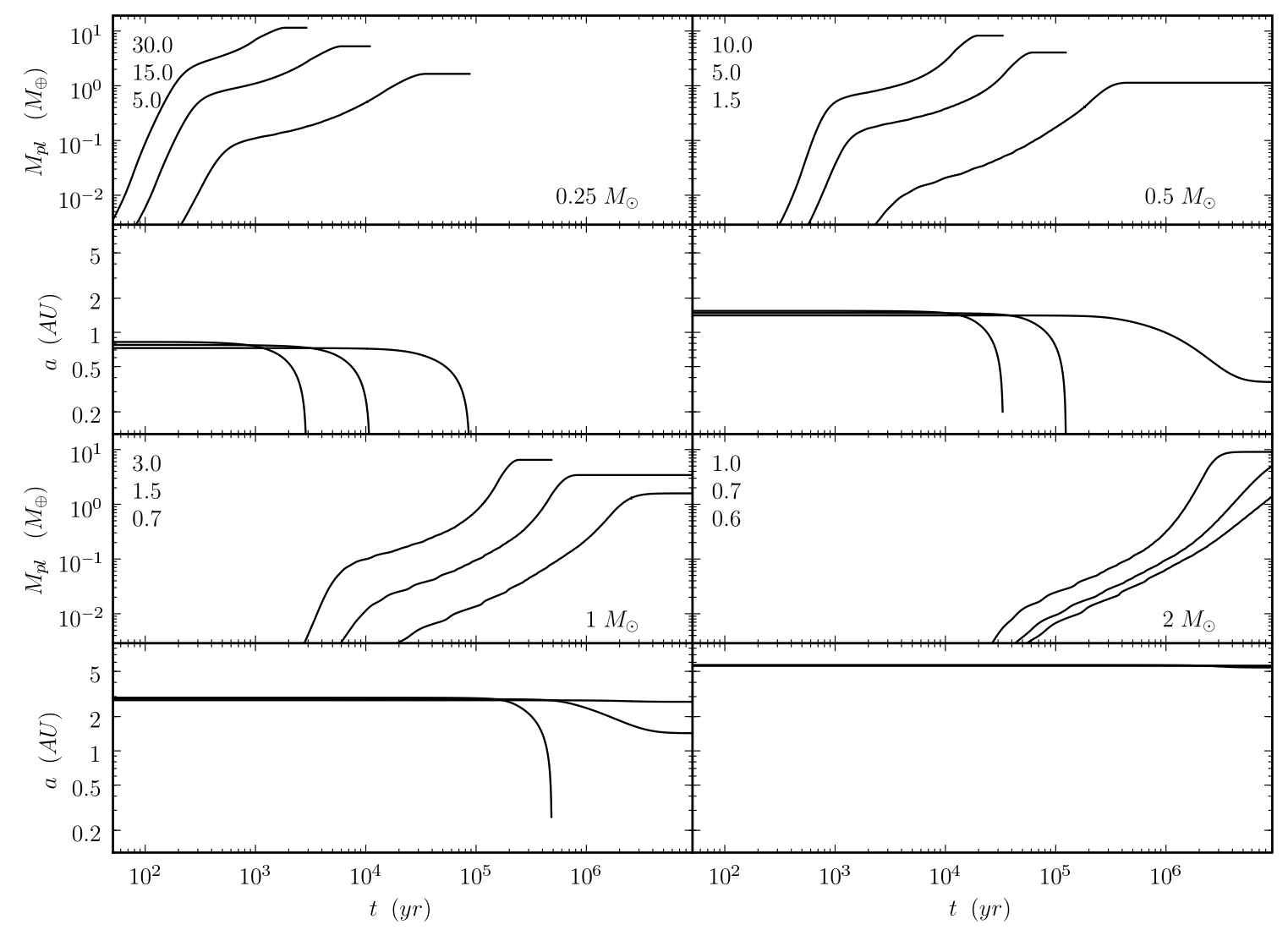

FIG. 6. - Same as Figure 5, but with $r=10 \mathrm{~km}$. Protoplanets still reach 1-10 $M_{\oplus}$, but require much higher disk masses. Thus, results for protoplanets migrating to the inner disk edge are similar to the $r=100 \mathrm{~m}$ case.

ward. Once scattered, the outer objects slowly migrate inward. For 0.25 and $0.5 M_{\odot}$ the scattered planets are still relatively close to the star, and have time to set up chains of (mostly first order) resonant orbits. A few collisions occur. For $1 M_{\odot}$ more objects are scattered outward, which continue chaotic growth. Despite the initial disruption by the migrating object, $\sim$ Earth-mass planets still form at $\sim 1$ AU.

When the migrating protoplanet encounters objects that have reached stable orbits, it shepherds them inward. These smaller objects accrete others as their orbits are pushed together, and several $\sim 1 M_{\oplus}$ rocky objects form. Shepherded objects may be accreted by the large migrator, or remain in interior resonant orbits (see Terquem \& Papaloizou 2007).

These simulations show that as with the gas giant case (Zhou et al. 2005), the effect of super-Earth migration on interior objects has observational consequences. Planets near the outer edge of the terrestrial region are scattered outward, while those in the inner region are shepherded to smaller radii. Shepherding results in multiple shortperiod planets with different compositions.

\section{DISCUSSION AND SUMMARY}

We have considered two scenarios for forming shortperiod $\lesssim 10 M_{\oplus}$ planets over a range of stellar masses: planet-planet scattering and type I migration.

Our models form planets in cold finger disks. These disks have large snow line enhancements compared to the MMSN model (Stevenson \& Lunine 1988;
Cuzzi \& Zahnle 2004). Water vapour from the terrestrial region condenses into ices outside the snow line as the gas disk diffuses and advects. The enhancement is increased by new water vapour delivered inside the snow line by drifting icy planetesimals (Cuzzi \& Zahnle 2004). Protoplanets forming in the cold finger regions near the snow line are much larger than others elsewhere in the disk.

We test the effectiveness of planet-planet scattering with $n$-body simulations. We consider stars with masses $M_{\star}=0.25-2 M_{\odot}$ and $10 M_{\oplus}$ planets. Planets with orbits near the limits of stability are evolved until a collision or ejection occurs, or $1 \mathrm{Gyr}$. Although equal mass planetplanet scattering can produce planets with small periastra for the lowest mass stars (Fig. 1), long circularisation times prevent them from achieving circular orbits on reasonable timescales. Thus, scattering is probably not a viable scenario for placing low-mass planets on shortperiod orbits for any stellar mass. For $0.25 M_{\odot}$, planets have periastra $\sim 0.05 \mathrm{AU}$ and semi-major axes $\sim 0.5 \mathrm{AU}$. Though transit durations are still several hours, orbital periods of several hundred days and maximum radial velocities of a few $\mathrm{m} / \mathrm{s}$ make these planets hard to detect.

Migration of icy protoplanets from the snow line is a viable mechanism for forming short-period super-Earths. Planet masses set whether they migrate to the inner disk edge before the gas disk disperses. Some planets with insufficient masses are stranded at intermediate radii as the gas disk disperses; a way to form "ocean planets" (Kuchner 2003; Léger et al. 2004). The minimum pro- 


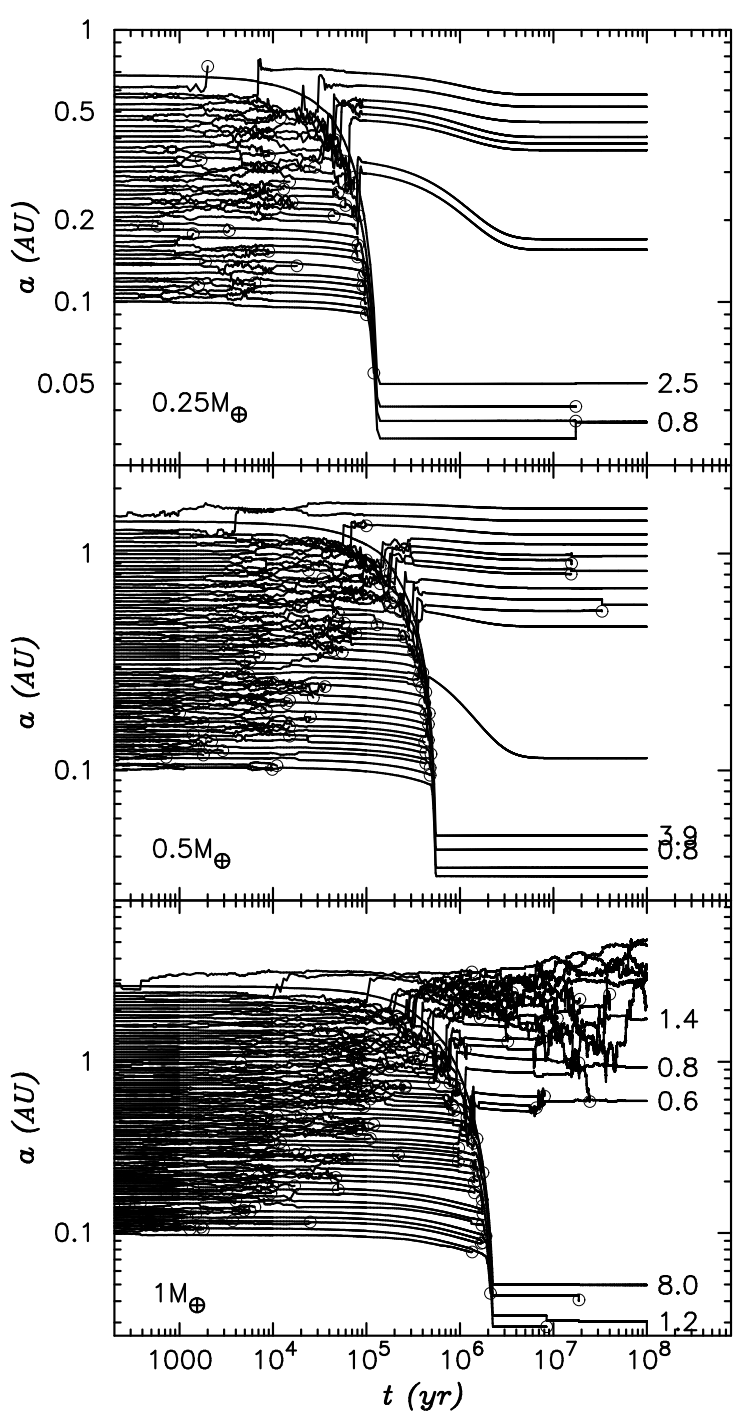

FIG. 7.- Migration of protoplanets in a cold finger disk for 0.25 , 0.5 , and $1 M_{\odot}$ (left to right). Initial outer planet masses are $2,3.2$, and $6 M_{\oplus}$. Lines end in a $\circ$ when a collision occurs. Surviving planets more massive than $1 M_{\oplus}$ are labelled by their final masses (in $M_{\oplus}$ ). Planets migrate earlier for lower stellar masses, because the snow line distance is closer. Objects scattered outward subsequently migrate, and some resume chaotic growth. Shepherded objects merge as their orbits are pushed together by the larger migrating protoplanet.

toplanet mass for migration to a close-in orbit increases as the snow line moves out with increasing stellar mass (Fig. 2). The maximum planet mass is $\sim 10 M_{\oplus}$, because above this mass they instead accrete large atmospheres and form gas giants.

Above $\sim 1 M_{\odot}$, the only protoplanets massive enough to migrate to close-in orbits are $\gtrsim 10 M_{\oplus}$ and no hot super-Earths form. This maximum stellar mass is independent of the disk mass distribution, and probes type I migration efficiency. Other uncertain parameters, such as snow line distance and disk profile do not have major observable consequences, but are not easily constrained by observations either.

For disks with similar snow line enhancements, the theory yields trends with metallicity (Fig. 3). For disk masses distributed as a power law, the frequency of short- period planets increases with metallicity, because most disks have low masses. However, if disk masses are distributed around a relatively high mass, planet frequency decreases with increasing metallicity, because planets forming in the most common disks are pushed above the gas accretion mass at high metallicities. As planetesimal size increases, growth slows, and becomes longer than the migration timescale. Simulations of concurrent accretion and migration with increased planetesimal sizes require much higher disk masses to yield similar results.

As icy planets migrate from the snow line, they interact dynamically with interior rocky protoplanets (Fig, 7). Protoplanets undergoing chaotic growth are scattered onto exterior orbits. Closer protoplanets on stable orbits damped by disk interaction are shepherded inward, and coalesce into a few rocky objects with masses $\sim 1 M_{\oplus}$. These objects may be accreted by the large migrating planet, or remain as separate planets on interior orbits. These orbits are likely near-commensurate with the icy migrators orbit (Terquem \& Papaloizou 2007). If planetary systems in such configurations are found in transit surveys, compositional models may discern differences, thus confirming their origins in rocky or icy regions (Valencia et al. 2007). However, different structural models may be degenerate if the planets have atmospheres (Adams et al. 2007).

Some planets may accrete hydrogen atmospheres due to a decreased planetesimal accretion rate following isolation (Ikoma et al. 2000; Rafikov 2006). To be observed as hot super-Earths requires subsequent photoevaporation (e.g. Baraffe et al. 2005). Significant photoevaporation of planets with massive atmospheres is unlikely unless the planet mass is in the $\lesssim 70 M_{\oplus}$ type I migration regime (Raymond et al. 2007). Thus, planets with remnant hydrogen atmospheres may form by the same migration mechanism we present here. Scattering is also a possibility for these planets to reach short-period orbits, because they have higher initial masses.

For planets originating in icy regions, their largely volatile composition has important implications for their evolution during and after formation. Icy grains may enhance growth if they stick together more easily, but also allows the possibility of large evaporation events in high energy collisions of larger objects. During the violent accretion process, and with the possible outcome of short-period orbits, melting and evaporation of ices will affect these planets (e.g. Lunine \& Stevenson 1982; Kuchner 2003; Selsis et al. 2007).

After migrating to close-in orbits, initially icy/watery planets may retain large super-critical steam atmospheres, or become rocky cores stripped of volatiles entirely. Kuchner (2003) considered the existence of volatile-rich planets in the Solar habitable zone, and suggested that planets around Solar luminosity stars would be safe from evaporation at $\gtrsim 1$ AU but not at closer distances. He also noted that lower EUV luminosities for M dwarfs makes these stars less likely to evaporate planetary atmospheres. More recently, Selsis et al. (2007) revisited the issue, and concluded that planets $\gtrsim 6 M_{\oplus}$ will retain most of their water content at $\gtrsim 0.04 \mathrm{AU}$ from a Solar-type star. The results of both studies suggest the evaporation timescale is strongly dependent on semimajor axis. Therefore, a trend may be noticeable within the small semi-major axis range of transiting planets. 
The picture that emerges is of systems with evaporated rocky planets inside $\sim 0.04 \mathrm{AU}$, and steam planets somewhat outside this distance. A few stalled ocean (Kuchner 2003; Léger et al. 2004) and icy planets extend through and past the habitable zone. For these planets, microlensing provides sensitivity complementary to transit and radial velocity methods at $\sim \mathrm{AU}$ distances (e.g. Beaulieu et al. 2006), which will help yield trends with semi-major axis, particularly for low-mass stars.

Surveys such as the MEarth Project (Nutzman \& Charbonneau 2007), CoRoT (Baglin 2003), and Kepler (Borucki et al. 2003) hope to discover super-Earths by the transit method. Like those discovered by radial velocity, most planets will orbit close to their parent stars. Because they are unlikely to form in situ, these planets necessarily require some form of migration or scattering from their formation regions. Observed systems will thus test and inform mechanisms that form and bring planets to visible orbits.

We acknowledge support from an Australian Postgraduate Award, a Smithsonian Astrophysical Observatory pre-doctoral fellowship (GK), and the NASA Astrophysics Theory Program through grant NAG5-13278 and the TPF Foundation Science Program though grant NNG06GH25G (SK). We thank the anonymous referee for a prompt report, which improved the content of the paper. $N$-body simulations were run on computers maintained by the RSAA Computer Section at Mt Stromlo Observatory.

\section{REFERENCES}

Adams, E. R., Seager, S., \& Elkins-Tanton, L. 2007, ArXiv e-prints, 710 [ADS]

Andrews, S. M. \& Williams, J. P. 2005, ApJ, 631, 1134 [ADS]

-. 2007, ApJ, 671, 1800 [ADS]

Asplund, M., Grevesse, N., \& Sauval, A. J. in , Astronomical Society of the Pacific Conference Series, Vol. 336, Cosmic Abundances as Records of Stellar Evolution and

Nucleosynthesis, ed. T. G. Barnes, IIIF. N. Bash, 25-+ [ADS]

Baglin, A. 2003, Advances in Space Research, 31, 345 [ADS]

Baraffe, I., Chabrier, G., Barman, T. S., Selsis, F., Allard, F., \& Hauschildt, P. H. 2005, A\&A, 436, L47 [ADS]

Beaulieu, J.-P. et al. 2006, Nature, 439, 437 [ADS

Bonfils, X., Forveille, T., Delfosse, X., Udry, S., Mayor, M., Perrier, C., Bouchy, F., Pepe, F., Queloz, D., \& Bertaux, J.-L. 2005, A\&A, 443, L15 [ADS]

Bonfils, X. et al. 2007, A\&A, 474, 293 [ADS]

Borucki, W. J. et al. in, Presented at the Society of Photo-Optical Instrumentation Engineers (SPIE) Conference, Vol. 4854, Future EUV/UV and Visible Space Astrophysics Missions and Instrumentation. Edited by J. Chris Blades, Oswald H. W. Siegmund. Proceedings of the SPIE, Volume 4854, pp. 129-140 (2003)., ed. J. C. BladesO. H. W. Siegmund, $129-140[\mathrm{ADS}]$

Brunini, A. \& Benvenuto, O. G. 2008, Icarus, 194, 800 [ADS]

Brunini, A. \& Cionco, R. G. 2005, Icarus, 177, 264 [ADS]

Chambers, J. 2006a, Icarus, 180, 496 [ADS]

Chambers, J. E. 1999, MNRAS, 304, 793 [ADS]

-. 2001, Icarus, 152, 205 [ADS]

-. 2006b, ApJ, 652, L133 [ADS]

Ciesla, F. J. \& Cuzzi, J. N. 2006, Icarus, 181, 178 [ADS]

Cresswell, P. \& Nelson, R. P. 2006, A\&A, 450, 833 [ADS]

Cuzzi, J. N. \& Zahnle, K. J. 2004, ApJ, 614, 490 [ADS]

Daisaka, J. K., Tanaka, H., \& Ida, S. 2006, Icarus, 185, 492 [ADS]

D'Angelo, G., Henning, T., \& Kley, W. 2002, A\&A, 385, 647 [ADS]

D'Angelo, G., Kley, W., \& Henning, T. 2003, ApJ, 586, 540 [ADS]

Durisen, R. H., Boss, A. P., Mayer, L., Nelson, A. F., Quinn, T., \& Rice, W. K. M. Protostars and Planets V, ed.

B. ReipurthD. Jewitt \& K. Keil, 607-622 [ADS]

Fischer, D. A. \& Valenti, J. 2005, ApJ, 622, 1102 [ADS]

Fogg, M. J. \& Nelson, R. P. 2007, A\&A, 472, 1003 [ADS]

Ford, E. B. \& Rasio, F. A. 2007, ArXiv Astrophysics e-prints [ADS]

Gillon, M., Pont, F., Demory, B.-O., Mallmann, F., Mayor, M., Mazeh, T., Queloz, D., Shporer, A., Udry, S., \& Vuissoz, C. 2007, A\&A, 472, L13 [ADS]

Gladman, B. 1993, Icarus, 106, 247 [ADS]

Goldreich, P., Lithwick, Y., \& Sari, R. 2004, ApJ, 614, 497 [ADS]

Goldreich, P. \& Soter, S. 1966, Icarus, 5, 375 [ADS]

Goldreich, P. \& Tremaine, S. 1980, ApJ, 241, 425 [ADS]

Goldreich, P. \& Ward, W. R. 1973, ApJ, 183, 1051 [ADS]

Haisch, Jr., K. E., Lada, E. A., \& Lada, C. J. 2001, ApJ, 553, L153 [ADS]

Hayashi, C. 1981, Progress of Theoretical Physics Supplement, 70,35 [ADS]
Ida, S. \& Lin, D. N. C. 2004, ApJ, 616, 567 [ADS]

-. 2005, ApJ, 626, 1045 [ADS]

—. 2008, ApJ, 673, 487 [ADS]

Ikoma, M., Nakazawa, K., \& Emori, H. 2000, ApJ, 537, 1013 [ADS]

Inaba, S. \& Ikoma, M. 2003, A\&A, 410, 711 [ADS]

Inaba, S., Tanaka, H., Nakazawa, K., Wetherill, G. W., \& Kokubo, E. 2001, Icarus, 149, 235 [ADS]

Johnson, J. A., Butler, R. P., Marcy, G. W., Fischer, D. A., Vogt, S. S., Wright, J. T., \& Peek, K. M. G. 2007, ApJ, 670, 833 [ADS]

Kennedy, G. M. \& Kenyon, S. J. 2008, ApJ, 673, 502 [ADS]

Kenyon, S. J. \& Bromley, B. C. 2006, AJ, 131, 1837 [ADS]

Kokubo, E. \& Ida, S. 1996, Icarus, 123, 180 [ADS]

-. 1998, Icarus, 131, 171 [ADS]

Kuchner, M. J. 2003, ApJ, 596, L105 [ADS]

Küppers, M. et al. 2005, Nature, 437, 987 [ADS]

Lee, M. H. \& Peale, S. J. 2002, ApJ, 567, 596 [ADS]

Léger, A., Selsis, F., Sotin, C., Guillot, T., Despois, D., Mawet, D., Ollivier, M., Labèque, A., Valette, C., Brachet, F., Chazelas, B., \& Lammer, H. 2004, Icarus, 169, 499 [ADS]

Lin, D. N. C., Bodenheimer, P., \& Richardson, D. C. 1996, Nature, 380, 606 [ADS]

Lissauer, J. J. 1987, Icarus, 69, 249 [ADS]

Lunine, J. I. \& Stevenson, D. J. 1982, Icarus, 52, 14 [ADS]

Masset, F. S., D'Angelo, G., \& Kley, W. 2006a, ApJ, 652, 730 [ADS]

Masset, F. S., Morbidelli, A., Crida, A., \& Ferreira, J. 2006b, ApJ, 642, 478 [ADS]

Muzerolle, J., Luhman, K. L., Briceño, C., Hartmann, L., \& Calvet, N. 2005, ApJ, 625, 906 [ADS]

Natta, A., Grinin, V., \& Mannings, V. 2000, Protostars and Planets IV, 559 [ADS]

Nelson, R. P. \& Papaloizou, J. C. B. 2004, MNRAS, 350, 849 [ADS]

Nutzman, P. \& Charbonneau, D. 2007, ArXiv e-prints, 709 [ADS]

Ohtsuki, K., Stewart, G. R., \& Ida, S. 2002, Icarus, 155, 436 [ADS]

Papaloizou, J. C. B. \& Larwood, J. D. 2000, MNRAS, 315, 823 [ADS]

Papaloizou, J. C. B., Nelson, R. P., Kley, W., Masset, F. S., \& Artymowicz, P. Protostars and Planets V, ed. , B. ReipurthD. Jewitt \& K. Keil, 655-668 [ADS]

Pollack, J. B., Hubickyj, O., Bodenheimer, P., Lissauer, J. J., Podolak, M., \& Greenzweig, Y. 1996, Icarus, 124, 62 [ADS]

Press, W. H., Teukolsky, S. A., Vetterling, W. T., \& Flannery, B. P. 1992, Numerical recipes in C. The art of scientific computing (Cambridge: University Press, —c1992, 2nd ed.) [ADS]

Rafikov, R. R. 2004, AJ, 128, 1348 [ADS]

—. 2006, ApJ, 648, 666 [ADS]

Rasio, F. A. \& Ford, E. B. 1996, Science, 274, 954 [ADS]

Raymond, S. N., Barnes, R., \& Mandell, A. M. 2007, ArXiv e-prints, 711 [ADS] 
Safronov, V. S. 1969, Evoliutsiia doplanetnogo oblaka. (1969.) [ADS]

Selsis, F. et al. 2007, Icarus, 191, 453 [ADS]

Stevenson, D. J. \& Lunine, J. I. 1988, Icarus, 75, 146 [ADS]

Stewart, G. R. \& Wetherill, G. W. 1988, Icarus, 74, 542 [ADS]

Tanaka, H., Takeuchi, T., \& Ward, W. R. 2002, ApJ, 565, 1257 [ADS]

Terquem, C. \& Papaloizou, J. C. B. 2007, ApJ, 654, 1110 [ADS]

Thommes, E. W., Duncan, M. J., \& Levison, H. F. 2003, Icarus, 161,431 [ADS]

Tremaine, S. \& Zakamska, N. L. in , American Institute of Physics Conference Series, Vol. 713, The Search for Other Worlds, ed. S. S. HoltD. Deming, 243-252 [ADS]
Udry, S., Fischer, D., \& Queloz, D. Protostars and Planets V, ed. B. ReipurthD. Jewitt \& K. Keil, 685-699 [ADS]

Valencia, D., Sasselov, D. D., \& O'Connell, R. J. 2007, ApJ, 665, 1413 [ADS]

Ward, W. R. 1997, Icarus, 126, 261 [ADS]

Weidenschilling, S. J. 1977, Ap\&SS, 51, 153 [ADS]

—. 2000, Space Science Reviews, 92, 295 [ADS]

Zhou, J.-L., Aarseth, S. J., Lin, D. N. C., \& Nagasawa, M. 2005, ApJ, 631, L85 [ADS] 